\title{
Vinculación Universidad - Sociedad: espacio para generar creatividad e innovación
}

\section{Linkage University - Society: space to generate creativity and innovation}

\author{
Pablo Fernando Cisneros Quintanilla ${ }^{1 *}$ y Karina Luzdelia Mendoza Bravo ${ }^{2}$ \\ ${ }^{1}$ Universidad Católica de Cuenca \\ ${ }^{2}$ Universidad Técnica de Manabí \\ *pcisneros@ucacue.edu.ec
}

DOI: https://doi.org/10.26871/killkana_social.v2i2.304

\begin{abstract}
Resumen
La proyección de la Universidad hacia la Sociedad adquiere su significativa relevancia en la búsqueda de una Educación Superior y con responsabilidad social. En el marco de una Educación Superior pertinente y con responsabilidad social, la proyección de la Universidad hacia la Sociedad adquiere una significativa relevancia. La Universidad ecuatoriana de manera general, inmersa en este proceso, tiene como misión preservar, desarrollar y promover, a través de sus procesos sustantivos y en estrecho vínculo con la sociedad, la cultura, lo que le permitirá formar profesionales integrales que contribuyan al desarrollo de la localidad, la provincia y el país. Sin embargo, se ha evidenciado que durante el proceso de formación de grado del profesional, existe una deficiente articulación de la Extensión Universitaria con los demás procesos sustantivos de la Universidad, identificándose además como únicos beneficiarios a los actores comunitarios y no a los estudiantes universitarios demostrándose así la ausencia del análisis de la extensión universitaria desde su dimensión pedagógica, donde se rescata el valor educativo que tiene la extensión universitaria en la formación de grado del profesional. En este contexto la extensión universitaria se constituye en una importante acción de vinculación con la comunidad y forma organizativa de aprendizaje, en respuesta a las demandas sociales, donde la universidad puede aportar y a la vez aprender de esa realidad. Sin embargo, la Extensión Universitaria como experiencia social y educativa no ha logrado una adecuada visibilidad y trascendencia en relación a su carácter formativo y de transformación social, lo que limita el alcance de este espacio formativo en el crecimiento personal, profesional y social de los estudiantes universitarios. Surge así la necesidad de resignificar las potencialidades de la Extensión Universitaria desde las prácticas extensionistas como espacio social de integración formación-experiencia-contexto comunitario, a lo cual esta investigación responde con el análisis tanto conceptual como experimental mediante el estudio de caso de una práctica extensionista realizada en universidades ecuatorianas, por parte de los autores del presente trabajo, orientada a la transformación de la práctica extensionista en experiencias formativas de compromiso social, integradas al proceso formativo del profesional.
\end{abstract}

Palabras clave: Aprendizaje en servicio, Dimensión Pedagógica, Extensión Universitaria, Práctica Extensionista, Vinculación con la Sociedad.

\section{Abstract}

The projection of the University towards Society acquires a significant relevance within the framework of a relevant higher education and with social responsibility. The Ecuadorian University in a general way, immersed in this process, has as its mission to preserve, develop and promote, through its substantive processes and in close connection with society, culture, which will allow it to train integral professionals that contribute to the development of the locality, the province and the country. However, it has become evident that, during the process of professional degree formation, there is a deficient articulation of the University Extension with the other substantive processes of the University, identifying as the only beneficiaries to the community actors and not to the university students. thus demonstrating the absence of the analysis of the university extension from its pedagogical dimension, where the educative value that the University Extension has in the professional degree training is recued. In this context, the university extension constitutes an important linking action with the community and an organizational form of learning, in response to social demands, where the university can respond and learn at the same time from the reality. However, the University Extension as a social and educational experience has not achieved adequate visibility and transcendence in relation to its formative nature and social transformation, which limits the scope of this training space in the personal, professional and social growth of students. university. Thus arises the need to resignify the potential of University Extension from the extension practices as a social space of integration training-experience-community context, to which this research responds with both conceptual and experimental analysis of an extension practice through a case study carried out in ecuadorian universities, by the authors of the present research, oriented to the transformation of the extensionist practice in formative experiences of social commitment, integrated to the formative process of the professional.

Key words: Service Learning, Pedagogical Dimension, University Extension, Extension Practice, Linkage with Society. 


\section{Introducción}

Los procesos formativos o sustantivos de la Educación Superior confluyen en las funciones de toda universidad, como son la docencia, la investigación y la extensión o vinculación con la colectividad. En todos y cada uno de esos procesos sustantivos se deben crear competencias creativas e innovadoras para los futuros profesionales.

En el presente caso se hace referencia de manera especial a la vinculación con la colectividad como uno de los espacios que permite crear competencias creativas e innovadoras en el ejercicio de su aprendizaje durante el servicio comunitario. Es decir, se refiere al aprendizaje en servicio.

Una de las características más importantes del conocimiento es su universalidad, por ello, los principios generales y los parámetros que se requieren tener en cuenta en todo sistema educativo, deberían estar establecidos con precisión en la normativa de cada país, empezando por la constitución y las leyes que lo rigen. (Mora, 2011)

Es necesario puntualizar que en los documentos normativos ecuatorianos, se utilizan indistintamente los términos vinculación con la colectividad, vinculación con la sociedad, o vinculación social, que es como se identifica en el Ecuador a lo que en otros países se denomina extensión universitaria. (Cisneros, 2017)

En el caso ecuatoriano, o más específicamente en las veinte constituciones que lo han regido y en las seis leyes de Educación Superior, se encuentra que la autonomía universitaria ha sido un elemento presente en todas ellas, sin embargo, por una extensiva y errónea interpretación de la autonomía universitaria, la universidad ecuatoriana ha permanecido por muchos años en una anarquía que ha condicionado la existencia de varios problemas entre los que se cuenta la situación caótica en la que se encontraban la extensión universitaria.

Con estos antecedentes se puede identificar que el principal problema estaba en la propia universidad, que no comprendía cuál era su misión y desvirtuó sus principios, funciones y procesos sustantivos, sin ninguna supervisión de organismos de control y amparada en un mal ejercicio de la autonomía universitaria.

La provisión de ciertos recursos económicos y, por sobre todo, la exigencia de la ley para enfrentar los procesos de evaluación y acreditación, obligaron a las instituciones de Educación Superior a invertir mayores esfuerzos en investigación científica y en extensión universitaria, que por supuesto mejoró respecto a épocas anteriores. Sin embargo, no se puede asegurar que esta respondió a la solución de los más importantes problemas que aquejaban a la sociedad sino que, por el contrario, era propuesta de manera desarticulada a la realidad nacional perdiendo todo sentido de pertinencia.

En el caso específico de los programas de extensión universitaria, adolecían de una lógica fundamental para enfrentar sus distintas dimensiones, lo que incidió en que las Instiruciones de Edicación Superior (IES) asumieran la difusión del arte, la cultura y la filantropía como las únicas formas de vincularse con la sociedad.

En este escenario, la extensión universitaria, de manera general, en las universidades ecuatorianas, se ha venido manejando de una forma totalmente desarticulada con la formación y la investigación.

Por tanto, el papel de la Educación Superior de manera general no debe limitarse a la transmisión de conocimientos y técnicas por medio de la docencia y la investigación, sino que por el contrario debe preparar al profesional, a través de la vinculación con la sociedad, para adquirir protagonismo en la solución de los problemas sociales; esta es la nueva mirada de la vinculación con la sociedad.

\section{Desarrollo}

Una revisión a la literatura internacional sobre pertinencia social universitaria permite constatar una atención creciente en su relación con la responsabilidad y la vinculación social.

Según la UNESCO (1998), la pertinencia social de la universidad es definida como la capacidad de responder a las necesidades o problemas sociales. Por tanto, la responsabilidad social universitaria y la vinculación social son conceptos propios de la pertinencia de la universidad.

La dimensión social de la universidad, que tradicionalmente ha encontrado su expresión explícita, principalmente, en los espacios y las actividades que desarrolla en su entorno, debe estar imbuida por una actitud de servicio.

La noción de aprendizaje-servicio refleja este nuevo enfoque de la función de servicio, cada vez más extendida y con muchos ejemplos de buenas prácticas, y supone una forma alternativa de vinculación con la sociedad.

A través de la vinculación con la sociedad, la universidad puede determinar el origen, el alcance y la validez objetiva del conocimiento y de la producción de conocimiento, es decir, puede pensarse a sí misma, desde la experiencia social, pero va más allá de los límites de la experiencia. O lo que es lo mismo, a través de la vinculación con la sociedad, la universidad puede convertirse en una institución que, en la tarea de transferir y aplicar conocimiento a la sociedad, vincularse y comprometerse socialmente, aprende de la misma sociedad a la que sirve y a la que aplica su saber.

En tal sentido, se puede verificar que en la Constitución ecuatoriana vigente (2008) cuando se establece la finalidad del sistema de Educación Superior, se declaran las tres funciones de la universidad, aunque no se lo haga explícitamente.

Art. 350.- "El sistema de Educación Superior tiene como finalidad la formación académica y profesional con visión científica y humanista" (Docencia); "la investigación científica y tecnológica" (Investigación); 'la innovación, promoción, desarrollo y difusión de los saberes y las culturas; la construcción de soluciones para los problemas del país, en relación con los objetivos del régimen de desarrollo" (Vinculación con la Sociedad). 
En concordancia con la Constitución en el Art. 8 de la Ley Orgánica de Educación Superior (LOES, 2010), se mencionan los fines de la Educación Superior y en el literal h) se indica: "Contribuir en el desarrollo local y nacional de manera permanente, a través del trabajo comunitario o extensión universitaria".

Por otro lado, en el Art. 13 se establece de manera precisa las funciones del sistema de Educación Superior cuando en el literal a) manifiesta: "Garantizar el derecho a la Educación Superior mediante la docencia, la investigación y su vinculación con la sociedad, y asegurar crecientes niveles de calidad, excelencia académica y pertinencia”.

Consecuentemente, es importante considerar que en el Art. 87, se solicita a los estudiantes "acreditar servicios a la comunidad mediante prácticas o pasantías preprofesionales" como requisito previo a la obtención del título, lo que puede interpretarse como el espacio para la Vinculación con la Sociedad.

Lo anterior se complementa con el Art. 88 en el que se especifica que en el servicio a la comunidad se propenderá a "beneficiar a sectores rurales y marginados de la población, si la naturaleza de la carrera lo permite, o a prestar servicios en centros de atención gratuita".

Desde este punto de vista se puede identificar a ese servicio comunitario con la vinculación social que quedaría identificada como la expresión científica del quehacer profesional al servicio de los sectores menos favorecidos.

En el Reglamento de Régimen Académico (RRA, 2017) se dedica el Título VI a la Vinculación con la Sociedad y se la define como: "La vinculación con la sociedad hace referencia a los programas de educación continua, gestión de redes, cooperación y desarrollo, relaciones internacionales, difusión y distribución del saber que permitan la democratización del conocimiento y el desarrollo de la innovación social".

Si se revisa el Art. 94 del RRA se puede interpretar que la Vinculación social forma parte y por ende está supeditado a los programas de práctica preprofesional de manera subordinada, pues se manifiesta que: "Las actividades de servicio a la comunidad contempladas en los artículos 87 y 88 de la LOES serán consideradas como prácticas pre profesionales. Para el efecto, se organizarán programas y proyectos académicos que deberán ejecutarse en sectores urbano-marginales y rurales..."

Por lo anotado se puede inferir que la vinculación social debe ser asumida como un proceso educativo transformador en el que se identifica un conjunto de rasgos que van configurando un esquema referencial conceptual, con un modelo de Vinculación con la Sociedad desde la “perspectiva de la integralidad". Entre los elementos más relevantes se considera:

- La Vinculación con la Sociedad como función universitaria y proceso académico, integrado a la docencia y la investigación.

- Proceso educativo/formativo, transformador e integrador.
- Proceso de comunicación dialógica que vincula críticamente el saber académico con el saber popular, donde cada actor aporta y aprende.

- Espacio de aprendizaje, reflexión y acción social interdisciplinaria.

- Experiencia organizada para la trascendencia del acto educativo universitario en sociedad.

- Espacio de prácticas sociales compartidas y colaborativas para comprender e intervenir de manera creativa e innovadora en las situaciones identificadas como problemas sociales.

En estos elementos, que se han considerado representativos de la diversidad conceptual que acompaña la vinculación con la sociedad, se identifican dos postulados esenciales: a) su potencial pedagógico como proceso académico y educativo, en la formación del estudiante universitario desde una perspectiva integral y humanizadora; b) su contribución a los procesos de transformación social como función universitaria; lo que en su unidad, permite conceptualizar la vinculación con la sociedad en términos de proceso educativo transformador, para la solución de problemas sociales identificados, a través de una relación dialógica, crítica y reflexiva de aprendizaje universidadsociedad, en el que los actores universitarios y los actores sociales construyen su experiencia formativa.

Estos elementos identificados en las conceptualizaciones de referencia, y la consideración de la vinculación con la sociedad como proceso educativo transformador, evidencian cómo la marginalidad de la vinculación social en relación a las funciones de docencia e investigación, el carácter extracurricular de los proyectos de vinculación, y la limitada participación de la comunidad universitaria en diálogo con los actores sociales, comienza a transitar hacia propuestas innovadoras en un marco de integración de las funciones sustantivas en las prácticas educativas, y la incorporación de los proyectos de vinculación al currículo universitario, como espacios sociales de aprendizaje y formación de profesionales socialmente comprometidos, desde un diálogo interactivo donde cada actor aporta y aprende.

En la Declaración Final del XIII Congreso Latinoamericano de Extensión Universitaria (2015), se reconoció como un importante tema del debate científico, el aporte de la Extensión Universitaria como espacio de aprendizaje, reflexión y acción interdisciplinaria en interacción con la docencia y la investigación, corroborando su función formativa de profesionales socialmente responsables, con una conciencia crítica en la búsqueda de alternativas para la gestión del desarrollo local y humano.

Se apuesta por la articulación entre formación y responsabilidad social, lo que ha abierto un nuevo camino al debate sobre la función formativa de los proyectos de vinculación, unido a la revitalización de la dimensión pedagógica de la vinculación social, que en su integración a las dimensiones institucional, política, social y comunicacional, 
le confieren una gran riqueza conceptual y reconfiguran la Vinculación con la Sociedad.

La dimensión pedagógica y social de la Vinculación con la Sociedad aparece con una marcada presencia en un espacio de encuentro formación - experiencia - contexto real.

Desde la perspectiva pedagógica de la vinculación con la sociedad, se transita a diversas modalidades de su incorporación al currículo de formación, a través de programas y proyectos docentes, extradocentes e investigativos como experiencias de aprendizaje en contexto; valorando, conceptualizando y dimensionando a la comunidad como escenario de experiencias de enseñanza y aprendizaje, en la que los saberes y conocimientos académicos y científicos se ponen en juego con las problemáticas sociales, teniendo en cuenta sus complejidades y la diversidad de actores sociales participantes.

Esta mirada pedagógica y social de la vinculación con la sociedad, resignifica la necesidad de entrelazar la formación social y académica, a través de prácticas que contemplen los espacios sociales en vinculación con la formación de los estudiantes, en las que se integren formación, experiencia y contexto real. Lo que reafirma Menéndez al considerar las prácticas de vinculación social (...) "como un valioso recurso pedagógico, promoviendo una «actitud» crítica y comprometida en cuanto al rol que como profesional puede tener en la democratización del conocimiento y la generación de cambios sociales innovativos". (Menéndez, 2013, p.89)

Este análisis desde la dimensión pedagógica sostiene que la Vinculación Social Universitaria es educativa y transformadora de saberes académicos, sociales y experienciales, “... ella genera subjetividades e improntas personales y sociales a la vez que procesa saberes académicos y experienciales" (Coscarelli, 2010, p.2), lo que confiere al proceso de vinculación social nuevos sentidos, a partir de la significatividad conceptual (saberes que se construyen a partir del diálogo que se establece entre los actores universitarios y los actores sociales), experiencial (relación del conocimiento con la práctica en un contexto real de solución de un problema social) y afectiva (nuevos saberes se insertan en el sistema de motivos, propósitos, expectativas de los sujetos participantes, generando nuevos sentimientos, intereses, actitudes y valores) que adquiere como resultado de la interacción dialéctica entre formación, experiencia y contexto real.

Para que la vinculación con la sociedad sea formativa de compromiso social debe posibilitar la construcción de un espacio de experiencias y saberes compartidos entre actores sociales, que propicien la problematización, concientización, reflexión y cambio, la sensibilidad del estudiante universitario ante los problemas de su entorno, la capacidad de identificarlos, de buscar soluciones a los mismos, de transformar la realidad en el sentido del progreso, de la mejora social y la realización personal. Todo ello implica cambios y transformaciones no solo en el medio social, sino también para cada participante (desarrollo profesional, personal y social).

El presente artículo se basa en el análisis del componente de vinculación con la sociedad en instituciones de Educación Superior tanto públicas como particulares, de manera general, y en un estudio de caso sobre las prácticas extensionistas en las carreras de educación, de manera particular, por lo que se propone producir impactos en la calidad y pertinencia de la Educación en los distintos niveles formativos, mediante su efecto sobre el desempeño profesional docente, atendiendo a los estándares establecidos por el Ministerio de Educación.

El logro del objetivo general de la Educación Superior, producirá impactos en la calidad del proceso y resultados de la formación de los educandos en los distintos niveles de enseñanza.

El impacto de la educación universitaria es comprendido no solo como producto cuantificable en términos de eficiencia académica; sino además como proceso cualitativo, considerando las transformaciones que se van consolidando en los modos de actuación profesional de los maestros, a través de la reflexión crítica de la práctica pedagógica, la ejecución de acciones transformadoras y su evaluación, como expresión de una armónica unidad teoría práctica que caracteriza las asignaturas del plan de estudio.

Todo ello articulado a los objetivos, políticas y lineamientos del Plan Nacional del Buen Vivir, a la Misión y Visión de las instituciones de Educación Superior, declarados en la pertinencia de cada programa.

Esta dinámica académica proporcionará el espacio propicio para generar proyectos de vinculación con la sociedad durante el trabajo investigativo de los estudiantes, tributando de esta forma al desarrollo del sistema educativo.

Lograr profesionales preparados, posibilita un empleo más eficiente de los recursos disponibles y que la inversión realizada a lo largo de los estudios sea revertida en logros para sí mismo, su familia y la comunidad donde se desempeñen.

Para el desarrollo de las actividades específicas de vinculación social en el campo educativo de las instituciones de Educación Superior, en íntima relación con las de prácticas de aplicación y o experimentación junto con el aprendizaje autónomo de los estudiantes, se plantean algunas líneas entre las que pueden estar las siguientes líneas de vinculación con la sociedad:

- Diagnóstico de la Docencia e Innovación Educativa en las Instituciones de Educación Superior.

- Levantamiento e identificación de Buenas prácticas en Innovación Educativa para las Instituciones de Educación Superior.

- Intervención y apoyo en los campos de la Pedagogía y la Didáctica, desde la Innovación y el Emprendimiento en la Metodología, los Recursos y las Herramientas de apoyo a la Docencia para las Instituciones de Educación Superior. 
- Aplicación las tecnologías de la información y la comunicación como elementos innovadores en el proceso enseñanza aprendizaje de los centros educativos.

En el marco de las líneas declaradas se pueden contemplar las siguientes actividades de vinculación con la sociedad:

- Socializar, en las instituciones educativas y en las comunidades en que están ubicadas, los diagnósticos de la situación pedagógica en que se encuentran las instituciones desde la mirada de la docencia y la innovación educativa, presentando evidencias de su influencia en la formación de los estudiantes.

- Ofertar cursos de formación continua para docentes de diferentes materias en los cuales se les prepare en materias de emprendimiento e innovación educativa.

- Desarrollar talleres con los docentes de las instituciones educativas, con el propósito de capacitarlos sobre la utilización de métodos, técnicas, estrategias, recursos y herramientas innovadoras que favorezcan el proceso de enseñanza aprendizaje.

- Elaborar propuestas metodológicas para favorecer el emprendimiento y la innovación en el campo educativo.

- Ejecutar eventos relacionados con la actividad académica que permitan destacar la importancia de las tecnologías de información y comunicación, como elemento innovador en el proceso docente.

- Diseñar espacios en los que pueda aplicares la innovación tecnológica para el mejoramiento del proceso docente.

- Organizar en instituciones educativas y en las comunidades en que están situadas, la presentación de buenas prácticas en el plano de la pedagogía, la docencia y la innovación educativa.

- Desarrollar debates grupales, en instituciones educativas y en las comunidades en que están enclavadas, que conlleven a la reflexión sobre la importancia de la Docencia y la Innovación en el ámbito educativo.

Desde estas consideraciones, las diferentes actividades de vinculación con la sociedad de las Instituciones de Educación Superior, deben ejecutarse a través de programas y proyectos en los que se involucre a las áreas de Educación Contínua y de Capacitación Docente, contando con el apoyo del departamento, dirección o estamento encargado de la Innovación y el Emprendimiento.

En este caso, atendiendo a los planteamientos del aprendizaje experiencial y del aprendizaje en servicio, los ámbitos de aplicación de las líneas de vinculación son la sociedad radican en las instituciones de educación, dando prioridad a aquellas ubicadas en zonas urbano-marginales o que se encuentran en situación desfavorable para el ejercicio de su labor educativa, a partir de los convenios suscritos con las coordinaciones zonales del Ministerio de Educación.

Si tiene en consideración todo lo antes indicado, la propuesta radica en que estos lineamientos que permitirían generar competencias innovadoras desde el proceso de vinculación con la sociedad, puedan ser debatidos en los espacios académicos correspondientes, de tal manera que se puedan encontrar los espacios necesarios para que se proponga situaciones similares tanto en el ejercicio de la docencia como en los procesos de investigación.

La compleja realidad de la actualidad mundial, condicionada históricamente y signada por la globalización, las crisis económicas, profundas transformaciones sociales y otras realidades, demanda el reajuste en las políticas educativas y la jerarquización de los valores sociales en que estas se sustentan. Unido a ello, el desarrollo acelerado de la ciencia y la técnica, el impacto de las Tecnologías de la Información y la Comunicación (TIC) y paradójicamente, el crecimiento de problemas existenciales y medioambientales, determinan que cobre mayor valor la formación y el desarrollo del hombre que asegure su inserción y protagonismo en ese contexto.

En estas condiciones, la educación es un fenómeno complejo que no resiste una visión única de análisis. Como proceso social está imbricado por un conjunto de factores económicos, políticos, sociales y culturales que condicionan la vida del hombre en un medio social determinado, en un momento histórico específico.

\section{Conclusiones}

Todo lo anteriormente analizado reafirma que la educación constituye el pilar fundamental para propiciar la transformación permanente del hombre, al promover su desarrollo integral, de manera que posibilite su participación responsable y creadora en la vida social, asegure su crecimiento humano y logre un alto sentido de compromiso con su bienestar y el de los demás.

Las Instituciones de Educación Superior ecuatorianas no escapan a esta realidad. La formación de grado del profesional requiere que se desarrolle a través de prácticas en las que los actores conformen sus identidades tanto en el ámbito individual como en el social, por medio de la integración de los procesos sustantivos, de modo que contribuyan a la formación de profesionales críticos, creativos, deliberativos y éticos, y se potencie así el pensamiento complejo y sistémico necesario para enfrentar en mejores condiciones las crecientes tensiones y problemas derivados del objeto de la profesión de cada una de las carreras.

Esta necesidad exige refundar la universidad, desde posiciones pedagógicas actuales, creativas, innovadoras y renovadoras, y afrontar de manera prioritaria la responsabilidad de articular la formación de grado del profesional en los diferentes niveles organizativos de la institución, hasta el trabajo docente en el aula, de tal manera que se propicie un adecuado proceso formativo orientado a la mejora continua de la calidad en la formación de grado de los profesionales de la Universidad.

Los elementos teóricos y prácticos antes señalados permiten reconocer la presencia de una contradicción entre las nuevas exigencias a las universidades ecuatorianas y 
a sus claustros para la implementación del nuevo RRA y los procesos de evaluación y acreditación y las insuficiencias teóricas y metodológicas que están en la base de la formación de grado del profesional en el contexto de la universidad ecuatoriana, desde un punto de vista que oriente las acciones hacia la proyección universitaria partiendo de los conceptos de la creatividad, la innovación y el emprendimiento.

\section{Referencias Bibliográficas}

Batista, A. (2016). Estrategia metodológica de integración de procesos sustantivos universitarios: contribución de la extensión universitaria a la promoción de salud en la Universidad de La Habana (Tesis Doctoral no publicada). Universidad de La Ha.

Beltrán, J., Iñigo, E., y Mata, A. (2014). La responsabilidad social universitaria, el reto de su construcción permanente. Revista Iberoamericana de Educación Superior (RIES), V(14), 3-18. Descargado de http://ries.universia.net/index .php/ries/article/view/367

Chaves, P., Prieto del Campo, C., y Ramírez Gallegos, R. (2013). Crisis del capitalismo neoliberal, poder constituyente y democracia real. Traficantes de Sueños. Descargado de https://dialnet.unirioja.es/ servlet/articulo? codigo=4417884

Cisneros, P. (2017). Concepción Pedagógica de la Formación de Grado del Profesional en la Universidad Católica de Cuenca - Ecuador (Tesis Doctoral no publicada). Universidad de La Habana.

Coscarelli, M. R. (2010). Formación en el campo de la extensión universitaria. Extensión en $\operatorname{Red}(01)$, 7. Descargado de http://sedici.unlp.edu . ar/handle/10915/41249

Horruitiner, P. (2009). La Universidad Cubana. Modelo de Formación. La Habana: Editorial Universitaria.

Mendoza, K. (2018). Alternativa metodológica para la práctica extensionista Club de Inglés como experiencia formativa de compromiso social en la Universidad Técnica de Manabí - Ecuador (Tesis Doctoral no publicada). Universidad de La Habana.

Menéndez, G. (2013). Institucionalización de la extensión. Conceptualización y dimensiones de la extensión. En A. Camillioni (Ed.), Integración docencia y extensión. otra forma de enseñar y aprender (pp. 83-91). Santa Fe: Universidad Nacional del Litoral.

Mora, M. (2011). La Educación única vía hacia la igualdad. Manta: Universidad Laica Eloy Alfaro de Manabí.

Morales, E., Muñoz, I., y Hernández, M. (2012). Reforma universitaria en Venezuela: ¿Pertinencia o vincula- ción socia. CPU-e, Revista de Investigación Educativa, 15(julio-diciembre de 2012), 119-139. Descargado de http://www.redalyc.org/pdf/ 2831/283123579007.pdf

Rafaghelli, M. (2013). Dimensión pedagógica de la extensión. En A. Camillioni (Ed.), Integración docencia y extensión. otra forma de enseñar (pp. 22-37). Santa Fe: Universidad Nacional del Litoral. Descargado de http:// web10.unl. edu.ar:8080/colecciones / bitstream/handle/11185/7963/ extension_interior_pag_22_37.pdf

Ramírez, R. (2010). Transformar la universidad para transformar la sociedad. Quito: Secretaría Nacional de Planificación y Desarrollo - SENPLADES.

Ramírez, R. (2013). Tercera Ola de Transformación de la Educación Superior en Ecuador. Hacia la constitucionalización de la sociedad del buen vivir. En Crisis del capitalismo neoliberal, poder constituyente y democracia real (pp. 197-236). Madrid. Descargado de https://dialnet.unirioja.es/ servlet/articulo?codigo=4417884

Ramírez, R., y Minteguiaga, A. (2010). Transformaciones en la Educación Superior Ecuatoriana: Antecedentes y perspectivas futuras como consecuencias de la nueva constitución política. Educación Superior y Sociedad, 1(enero de 2010), 129-154. Descargado de http: / / unesdoc.unesco.org/images/ $0019 / 001917 / 191731 \mathrm{~m} \cdot \mathrm{pdf}$

Universidad Nacional Autónoma de México. Instituto de Investigaciones sobre la Universidad y la Educación, J., Íñigo-Bajo, E., y Mata-Segreda, A. (2014). Revista iberoamericana de educación superior. (Vol. V) (n. ${ }^{0}$ 14). Instituto de Investigaciones sobre la Universidad y la Educación. Descargado de http: / / www.redalyc.org/articulo . oa?id=299132095001

Recibido: 18 de junio de 2018

Aceptado: 29 de junio de 2018 